\title{
A simple approach of three-isocenter IMRT planning for craniospinal irradiation
}

\author{
Zheng Wang ${ }^{1 \dagger}$, Wei Jiang ${ }^{1 *+}$, Yuanming Feng ${ }^{2}$, Yang Guo ${ }^{1}$, Zheng Cong ${ }^{1}$, Bin Song ${ }^{1}$ and Yu Guo ${ }^{3}$
}

\begin{abstract}
Purpose: To develop a new IMRT technique to simplify the process and improve efficiency in radiotherapy treatment planning for craniospinal irradiation (CSI) treatment.

Methods: Image data of 9 patients who received CSI treatment in 2012 were used, the prescription was 36Gy in 20 fractions. Two treatment plans were created for each patient, one was with the new technique called three-isocenter overlap-junction (TIOJ) IMRT and the other was with the three-isocenter jagged-junction (TIJ) IMRT technique. The comparative study was conducted using the parameters of heterogeneity index $(\mathrm{HI})$, conformity index $(\mathrm{Cl})$, and doses to the organs at risk (OARs).

Results: Comparing the TIOJ IMRT plans with the TIJJ IMRT plans, the average homogeneity index is $0.071 \pm 0.003$ and $0.077 \pm 0.002$, respectively, and the averaged conformity number is $0.80 \pm 0.012$ and $0.80 \pm 0.009$, respectively. There are no significant differences $(p>0.05)$. Both plans provide satisfactory sparing for the OARs.

Conclusions: The TIOJ IMRT technique for CSI treatment planning can create similar plans as with the TIJJ IMRT technique, but the new technique greatly simplifies the steps required to manually set field widths and boundaries and improved efficiency.
\end{abstract}

Keywords: Craniospinal irradiation, Field junction, Field edge matching, Overlap-junction IMRT

\section{Introduction}

Craniospinal irradiation (CSI) has become an important treatment method for primary tumors. Commonly treated tumors include medulloblastoma, high-risk germcell tumors, and some radio-sensitive secondary malignant tumors of the meninges. Emerging radiotherapy techniques, such as three-dimensional conformal radiotherapy (3DCRT) and intensity modulated radiation therapy (IMRT), have gradually replaced the traditional large field radiotherapy technology used in CSI treatment. CSI involves complex anatomical structures and requires complex treatment planning, which often entails setting multiple isocenters and matching a large number of fields to obtain satisfactory plans. IMRT technology can offer better comformity Index (CI) and homogeneity index $(\mathrm{HI})$ than traditional multi-field 3DCRT in complex target areas. Inverse treatment planning with IMRT reduces the difficulty of planning

\footnotetext{
* Correspondence: weijianghh@163.com

${ }^{\dagger}$ Equal contributors

'Department of Radiation Oncology, Huanhu Hospital, Tianjin, China

Full list of author information is available at the end of the article
}

and implementation as well. These two advantages are particularly important in CSI. Helical tomotherapy $[1,2]$ and radiotherapy techniques based on protons [3] have also been used in CSI. Another emerging radiotherapy technique, volumetric modulated arc therapy (VMAT), has also been applied in CSI treatment $[4,5]$.

In comparison to other IMRT techniques [6-8], the three-isocenter jagged-junction (TIJJ) IMRT recently proposed by Cao et al. [9] achieves similar $\mathrm{CI}$ and $\mathrm{HI}$ and simplifies planning and implementation processes. Yet the treatment plan still involves adjustment of a large number of staggered fields, and the planning process is time consuming. Reducing the complexity of treatment plans and shortening treatment time will make the treatment more reliable and improve the overall treatment quality. For this purpose, we have developed a simplified IMRT technique called three-isocenter overlap-junction (TIOJ) IMRT, and presented in this manuscript. The goal is to simplify the implementation of the treatment plan, ensure satisfactory $\mathrm{CI}$ and $\mathrm{HI}$, and reduce the time needed for planning and implementing CSI treatment.

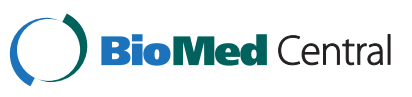




\section{Material and methods}

Image data of nine patients who received CSI treatment in 2012 were used for this study (Table 1). Two CSI treatment plans were made for each patient, one was TIOJ IMRT as defined in this manuscript and the other was TIJJ as defined by Cao et al. [9]. The two plans were compared using the parameters of $\mathrm{HI}, \mathrm{CI}$, and doses to the organs at risk (OARs).

Among the nine patients evaluated, three were averagerisk medulloblastoma patients who received reduced dose (23.4 Gy in 13 fractions) while the others received 36 Gy in 20 fractions. To make the plans comparable, we created the plans with the same prescription of 36 Gy in 20 fractions for all patients. The study using patient data was approved by the Ethics Committee of Tianjin Huanhu Hospital (\#2012-3).

\section{Patient position and simulation}

All patients were set up in a supine position with both hands naturally and comfortably placed at their sides. A thermoplastic facial mask and a body mask were used to fix the patient. After the immobilization, Six BrainLAB (BrainLAB AG, Feldkirchen, Germany) real-time infrared reflective marker balls were placed on the surface of the thermoplastic masks. The simulation CT images were acquired using a Brilliance CT Big Bore (Philips Medical Systems, Cleveland, OH, USA). Scan range was from the top of the head to the proximal femur, which included the entire torso and arms. Slice thickness was $3 \mathrm{~mm}$.

\section{Planning}

Target delineation: Delineation of planning target volume (PTV) and OARs were both based on CT images. PTV cns $_{\text {. }}$ included the brain (PTV brain $)$ and spinal cord ( $\left.\mathrm{PTV}_{\text {spinal }}\right)$. PTV brain included the whole brain, the meninges and $3 \mathrm{~mm}$ beyond their external boundary. PTV $\mathrm{P}_{\text {spinal }}$ included $\mathrm{C} 1$ through $\mathrm{S} 3$, and $5 \mathrm{~mm}$ beyond their external boundary. Lens, optic nerves, eyes, thyroid, heart, lungs, liver, and kidneys were delineated as OARs for comparison.

Table 1 Patient demographics

\begin{tabular}{lllll}
\hline Patient & Gender & Age $(\mathbf{y})$ & Diagnosis & PTV length $(\mathbf{c m})$ \\
\hline 1 & M & 8 & GCT & 62.3 \\
\hline 3 & M & 35 & MM & 78.6 \\
\hline 4 & M & 13 & GCT & 64.7 \\
\hline 5 & M & 14 & Ependymoma & 67.9 \\
\hline 6 & M & 26 & GCT & 70.8 \\
\hline 7 & F & 30 & MM & 76.2 \\
\hline 8 & F & 14 & GCT & 62.5 \\
\hline 9 & M & 10 & GCT & 68.3 \\
\hline Patent dem & F & 24 & MM & 75.6 \\
\hline
\end{tabular}

Patient demographics at time of CT scan acquisition.

Abbrevations: PTV Planning target volume, $M$ male, $F$ female, GCT Germ cell tumor, MM Medublastoma.
IMRT plans for all patients were generated using Eclipse treatment planning system (Eclipse TPS 10.0.24, Varian Medical Systems Inc., Palo Alto, CA, USA). A 6MV (Varian 6EX) linear accelerator equipped with a 120-leaf multileaf collimator (MLC) was used to implement the treatment plan. The BrainLAB ExacTrac was used for setup before treatment, moving the table for different isocenters and monitoring the mobility of the patient during treatment.

\section{TIOJ IMRT plan}

The three isocenters of the beams were placed in the TIOJ plan such that they had the same distance of $100 \mathrm{~cm}$ to the source, and were denoted as A, B, C, respectively. As shown in Figure 1a, the line connecting the three isocenters ( $\mathrm{A}, \mathrm{B}$ and $\mathrm{C}$ ) was parallel to and above the midline of the patient in the sagittal plane. All of the $\mathrm{PTV}_{\text {spinal }}$ were located dorsal to this connecting line. The positions of $\mathrm{A}, \mathrm{B}$ and $\mathrm{C}$ on this connecting line were determined by the following steps. Point A was set as the midpoint along the rostral-caudal direction of the $\mathrm{PTV}_{\text {brain }}$ in the sagittal plane. If the length of the PTV $\mathrm{Pns}_{\mathrm{cn}}$ was less than $80 \mathrm{~cm}, \mathrm{~B}$ and $\mathrm{C}$ were set to make $\mathrm{A}, \mathrm{B}$, and $C 25 \mathrm{~cm}$ apart to each other. If the length of the $\mathrm{PTV}_{\text {cns }}$ was greater than $80 \mathrm{~cm}, \mathrm{~B}$ and $\mathrm{C}$ were set to make A, B, and C $30 \mathrm{~cm}$ apart to each other. This was to ensure sufficient beam overlap among the fields with different isocenters. The collimator angle was set at $0^{\circ}$ for all the field sets. The fields in every field set of an isocenter had the same field size. With this set-up scheme, two beam overlap regions with lengths of $15 \mathrm{~cm}$ or $10 \mathrm{~cm}$ were formed between the three isocenters. The field set with isocenter A (IsoA) contained seven fields, and the gantry angles were $0^{\circ}, 65^{\circ}, 100^{\circ}, 123^{\circ}, 230^{\circ}, 257^{\circ}$ and $290^{\circ}$, respectively. The field sets with IsoB and IsoC had three radiation fields each, and the gantry angles were $145^{\circ}, 180^{\circ}$ and $215^{\circ}$, respectively. As the distance from spine to treatment machine head for these posterior and posterior oblique fields in the field sets for spinal cord treatment is shorter than $100 \mathrm{~cm}$, the leaf width of MLC projected in the spinal cord region is smaller than its nominal width, this could theoretically provide better homogenous dose distribution in the volumes of field junctions.

The constrains set for the inverse optimizations were as following, 99\% of the PTV (for both PTV brain and PTV $\mathrm{Ppinal}_{\text {) is to }}$ receive $95 \%$ of the prescribed dose, the doses to the lens and eyes are to be kept to the lowest achievable, and there are no dose constrains for other OARs.

\section{TIJJ IMRT plan}

The method first proposed by Cao et al. [9] is shown in Figure 1b. Plans covered the cranial and spinal PTVs with the use of three isocenters. Care was taken to ensure 

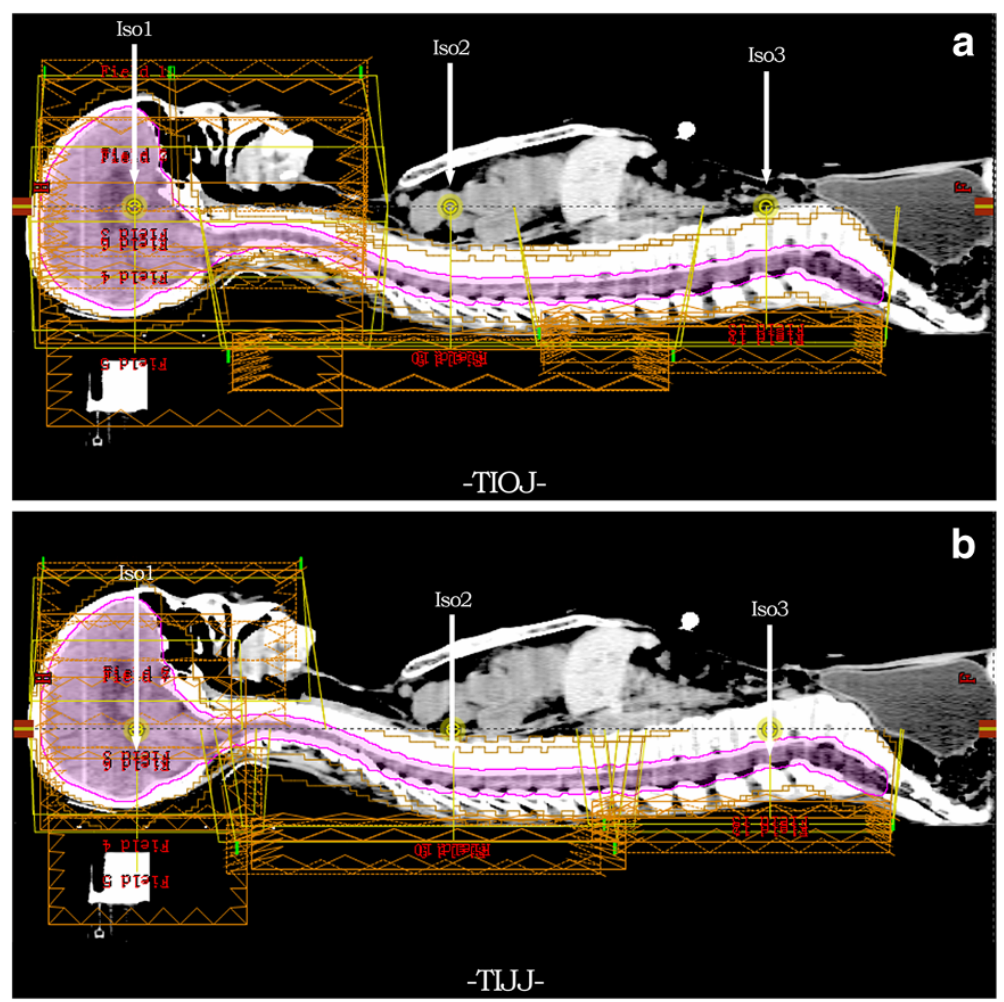

Figure 1 Beam arrangements for TIOJ plan and TIJ plan. Sagittal view shows beam arrangements for both cranial and spinal regions for TIOJ plan (a) and TIJ plan (b). The conventional plan figure shows the placement of isocenters in a TIOJ plan has beams with shorter source to surface distances (SSDs) which make the distances between the MLC and the spinal cord shorter.

that no beam entered through the patient's shoulders. One isocenter (Iso1) was placed in the cranial PTV, and two isocenters (Iso2 and Iso3) were placed in the spinal PTV, with Iso2 located superior to Iso3. The three isocenters were collinear, and were placed near the patient's midline. For the patients scanned in the supine position, the Iso1 field set consisted of seven fields with gantry angles of $0^{\circ}$, $65^{\circ}, 100^{\circ}, 123^{\circ}, 230^{\circ}, 257^{\circ}$, and $290^{\circ}$. Both field sets from Iso 2 and Iso3 consisted of three beams with gantry angles of $145^{\circ}, 180^{\circ}$, and $215^{\circ}$. The collimator angles were set at $0^{\circ}$ for all fields. Adjacent field sets were intentionally overlapped to treat a common region of the spine. Field edges were staggered in $1.1 \mathrm{~cm}$ steps. The lateral or nearly lateral field cannot treat through the shoulders.

\section{Plan evaluation}

The two plans were compared, mainly using $\mathrm{HI}$ and $\mathrm{CI}$ of the target areas. Currently there are multiple definitions for $\mathrm{HI}$ and CI. The definitions used by Cao et al. [9] were adopted in order to compare the two methods. HI is defined as

$$
H I=\frac{\mathrm{D}_{2 \%}-\mathrm{D}_{98 \%}}{\mathrm{D}_{\text {Median }}}
$$

where $D_{\text {Median, }} D_{2 \%}$ and $D_{98 \%}$ are doses received by $50 \%$, $2 \%$ and $98 \%$ the PTV volume, respectively. CI is defined as

$$
C N=\frac{V_{T \text { pres }}}{V T} \times \frac{V_{\text {Tpres }}}{V_{\text {pres }}}=\frac{V_{T \text { pres }}^{2}}{V T \times V_{\text {pres }}}
$$

where $V_{T}$ pres is the targe volume covered by $95 \%$ isodose surface. $V_{\text {pres }}$ is the treated volume covered by $95 \%$ isodose surface and VT is the volume of target. The value of $C I$ is in the range of $0-1$, where a value closer to 1 indicates better conformity. The value of $\mathrm{HI}$ is in the range of $1-0$, where a value closer to 0 indicates better homogeneity.

Plan quality assurance for the cranial-spinal and spinalspinal junctions

In complex plans, multiple isocentes can overlap. Homogeneity of dose distribution in overlapping radiation fields of different isocenters must be achieved. The influence of variation in treatment table positioning on dose distribution must be carefully evaluated. In this study, the plan quality assurance (QA) was conducted with an IBA matrix (IBA Dosimetry Germany) The QA plans for the IBA matrix were generated in Eclipse TPS, and the measurements of dose distributions in the overlapped 
areas of field junctions were performed afterwards. Due to limitations in the size of the IBA matrix test model $(27 \mathrm{~cm} \times 27 \mathrm{~cm})$, only dose distribution and pass rate in the overlapping areas were measured in each plan.

\section{Statistical analysis}

Statistical analysis was performed using the SPSS statistical analysis software package, Version 18.0 (SPSS Inc., Chicago, IL). A nonparametric related-samples Wilcoxon signed-ranks test was chosen because the sample sizes were small and not of a normalized distribution, P values $<0.05$ were considered statistically significant.

\section{Results}

For the nine patients included in this study, both TIOJ and TIJJ IMRT reach the goal of the 95\% isodose curve covering at least $99 \%$ of the PTV. Figure 2 shows the PTVcns coverage for patient 5 using a dose volume histogram (DVH). Both plans meet the initial goal for the target volume coverage.

PTVens coverage, $\mathrm{HI}, \mathrm{CI}$ and other results in the two plans are listed in Table 2. HI and CI obtained with TIOJ are $0.071 \pm 0.003$ (Mean \pm variance) and $0.80 \pm 0.012$, respectively. HI and CI obtained with TIJJ are $0.077 \pm$ 0.002 and $0.80 \pm 0.009$, respectively. Both results meet the PTV dose coverage requirements as published in the International Commission on Radiation Units (ICRU) guidelines $[10,11]$. There are no significant differences for $\mathrm{CI}$ and $\mathrm{HI}(\mathrm{P}>0.05)$.

Figure $3 \mathrm{a}$ and $\mathrm{b}$ shows the dose distribution in the two plans in patient 5. No "cold" dosing spots or "hot" dosing spots are found in the radiation beam overlapping regions between isocenters. The two treatment planning techniques provide similar plans in this regard.
Table 2 Evaluation parameters for TIOJ and TIJJ plans

\begin{tabular}{cccccc}
\hline Patient & \multicolumn{3}{c}{ HI } & & \multicolumn{2}{c}{ Cl } \\
\cline { 2 - 3 } \cline { 6 - 6 } & TIOJ & TIJ & & TIOJ & TIJ \\
\hline 1 & 0.083 & 0.086 & & 0.83 & 0.83 \\
\hline 2 & 0.072 & 0.072 & & 0.74 & 0.78 \\
\hline 3 & 0.070 & 0.070 & & 0.81 & 0.80 \\
\hline 4 & 0.083 & 0.085 & & 0.86 & 0.84 \\
\hline 5 & 0.061 & 0.063 & 0.80 & 0.78 \\
\hline 6 & 0.070 & 0.070 & 0.79 & 0.79 \\
\hline 7 & 0.061 & 0.060 & 0.81 & 0.81 \\
\hline 8 & 0.070 & 0.070 & 0.83 & 0.72 \\
\hline 9 & 0.069 & 0.081 & 0.76 & 0.69 \\
\hline Mean \pm variance & $0.071 \pm 0.003$ & $0.077 \pm 0.002$ & $0.80 \pm 0.012$ & $0.80 \pm 0.009$ \\
\hline Abbrevations: Cl conformity index, $H$ HI homogeneity index. &
\end{tabular}

The total monitor units (MUs) needed for delivering the fraction dose of 1.8 Gy are 1907.4 \pm 60.5 (ranging from 1575 to 2104 ) with TIOJ plan and $1903.3 \pm 34.8$ (ranging from 1575 to 2253) with TIJJ plan, respectively, there is no significant difference $(\mathrm{P}>0.05)$. The average doses to the torso in the two plans are $8.75 \pm 0.59$ Gy with TIOJ and $8.26 \pm 0.53$ Gy with TIJJ, respectively, there is no significant difference $(\mathrm{P}>0.05)$. No correlation between total $\mathrm{MU}$ and torso dose in either plan was found.

Figure 4 shows the doses to different OARs in the two plans. There are no significant differences in the doses of different OARs with the two plans $(\mathrm{P}>0.05)$.

The comparison of the dose distribution in spinal-spinal beam overlapping regions using an IBA matrix for patient 5 is shown in Figure 5. QA evaluations of TIOJ plans using Gamma index (3\% for absolute dose and $3 \mathrm{~mm}$ for relative dose evaluation) were performed. All plans has a pass rate above $90 \%$. The mean pass rate is $94.6 \%$ (ranging

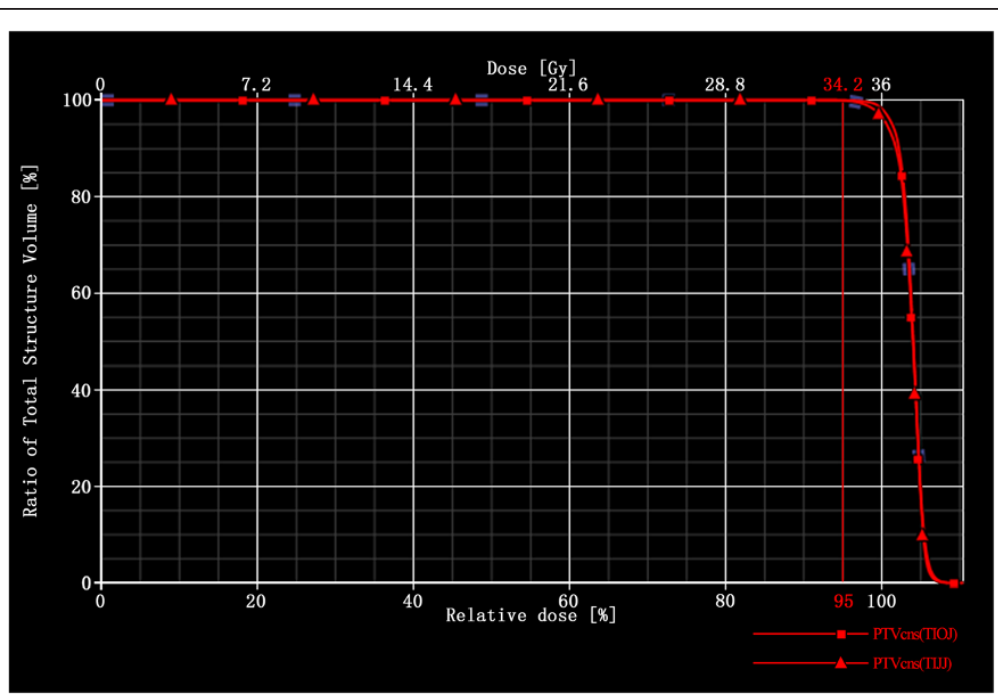

Figure 2 Dose volume histogram (DVH) comparison of target coverage. DVH of a representative plan comparing target coverage between the TIOJ plan and the TIJJ plan. The target coverage of both plans meet the initial goal for the target volume coverage. 

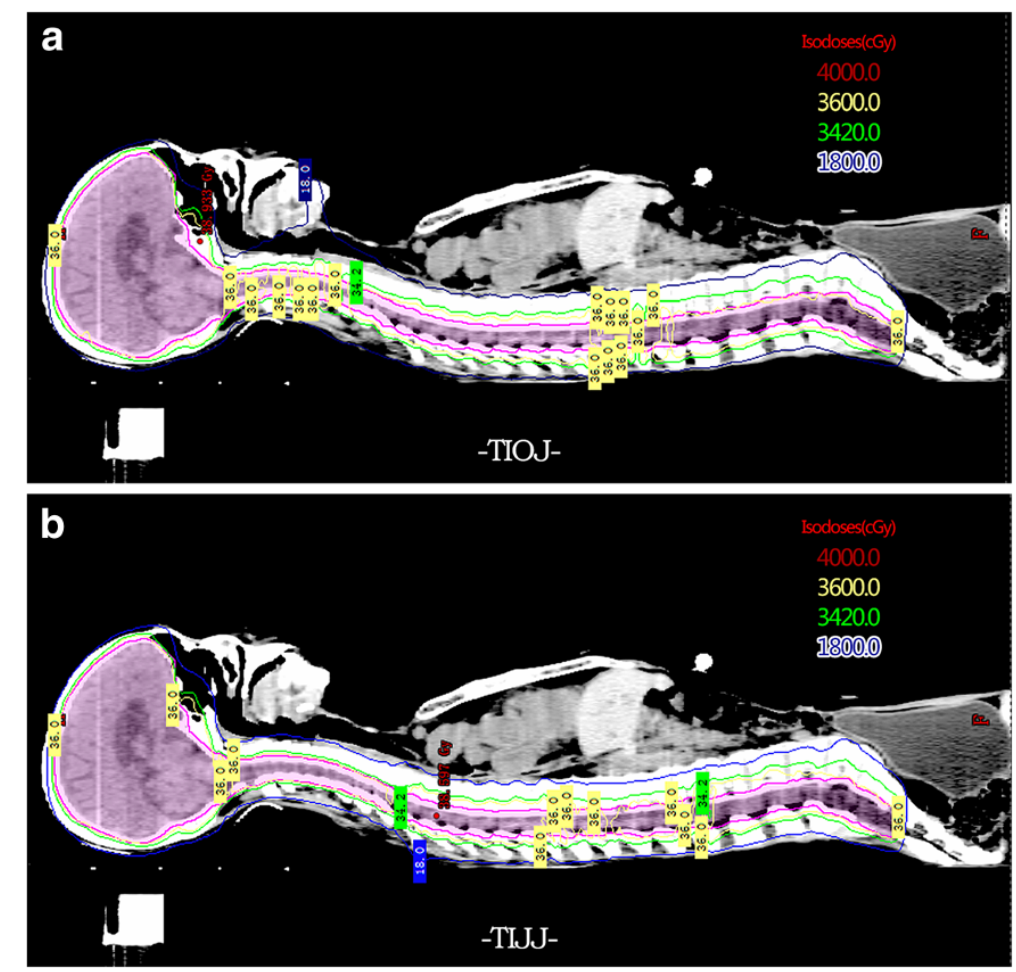

Figure 3 Isodose distribution of Patient 5 for TIOJ plan and TIJ plan. Isodose distribution on a midline sagittal CT slice of Patient 5 for TIOJ plan (a) and TIJJ plan (b). Color legend indicates isodose lines, in centigrays. Planning target volume is shown in pink.

from $92.5 \%$ to $97.7 \%)$. The measured and planned dose profiles agree well and there are no cold or hot spots.

\section{Discussion}

Simulation, planning, QA and treatment delivery processes for CSI techniques require great care. Patient position, patient immobilization, target delineation, protection of OARs, HI, and the field junctions can have serious effects on the treatment outcome. Compared to traditional $2 \mathrm{D}$ radiation therapies, 3D radiation technologies such as IMRT and VMAT [4,5] provide much

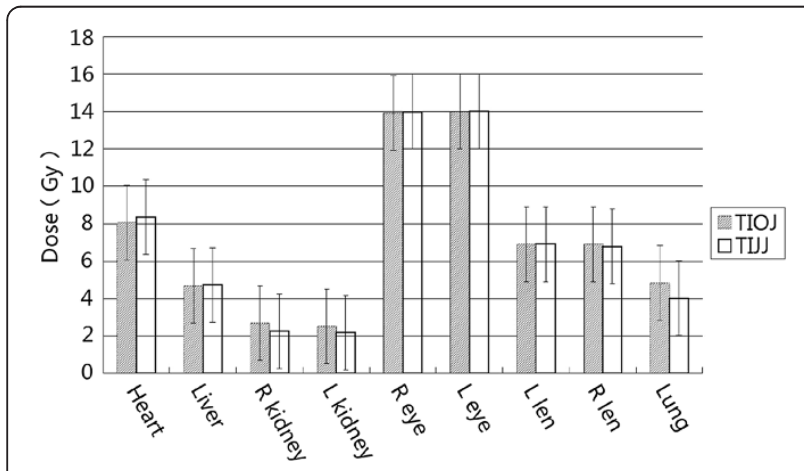

Figure 4 Median and variation. Median and variation (shown as bars) of mean doses to OARs in TIOG (shade) and TIJ (white) plans. L: Left; R: right. better target coverage and protection of OARs. Although there have been previous reports of successful applications of IMRT technology in CSI $[6,7,12,13]$, most of these only partially modified or optimized the traditional CSI plan by adding an IMRT component. The technical advantages of IMRT were not fully utilized. Recently, Cao et al. [9] simplified the radiation field matching issue involved in traditional planning by using a single IMRT plan with three isocenters. In practice, this technique requires large amounts of manual adjustment of radiation fields to generate beam overlapping regions which is time consuming.

The TIOJ IMRT technique presented in this manuscript is similar to the ones proposed by Seppala et al. [6] and Cao et al. [9]. But with TIOJ, there is no need for manual adjustment of radiation fields to generate beam overlapping regions and the difficulties in the planning process is greatly reduced while maintaining similar and satisfactory plan results.

As compared to the plan by Cao et al., the placement of isocenters in a TIOJ plan has beams with shorter source to surface distances (SSDs) which make the distances between the MLC and the spinal cord shorter. This makes the projected MLC width smaller in the spinal cord, which is equivalent to having thinner MLC leafs, which helps maximize the potential of the inverse treatment planning system and avoiding hot or cold 


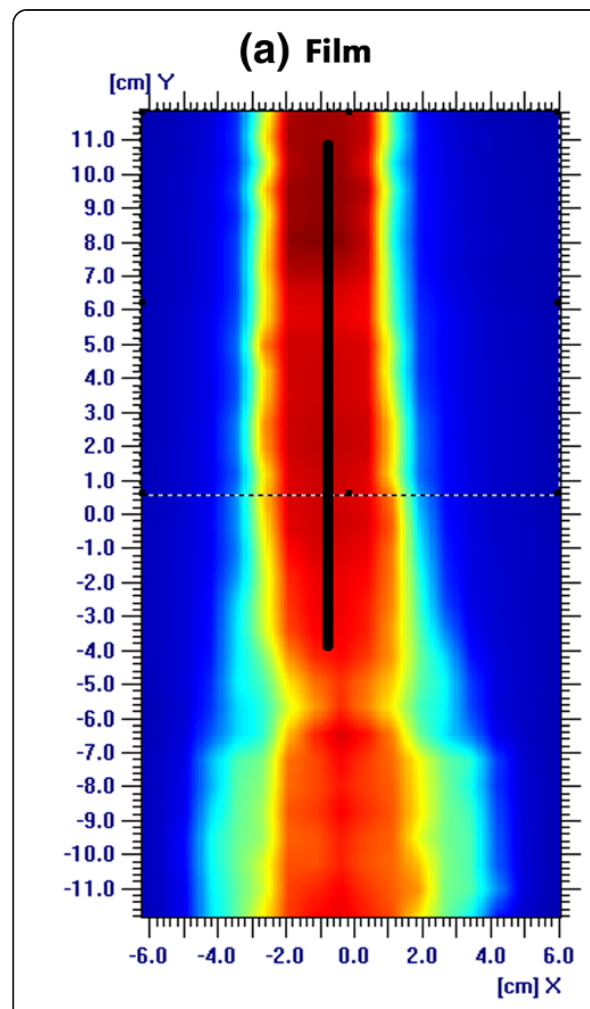

\section{(b) Gamma evaluation}

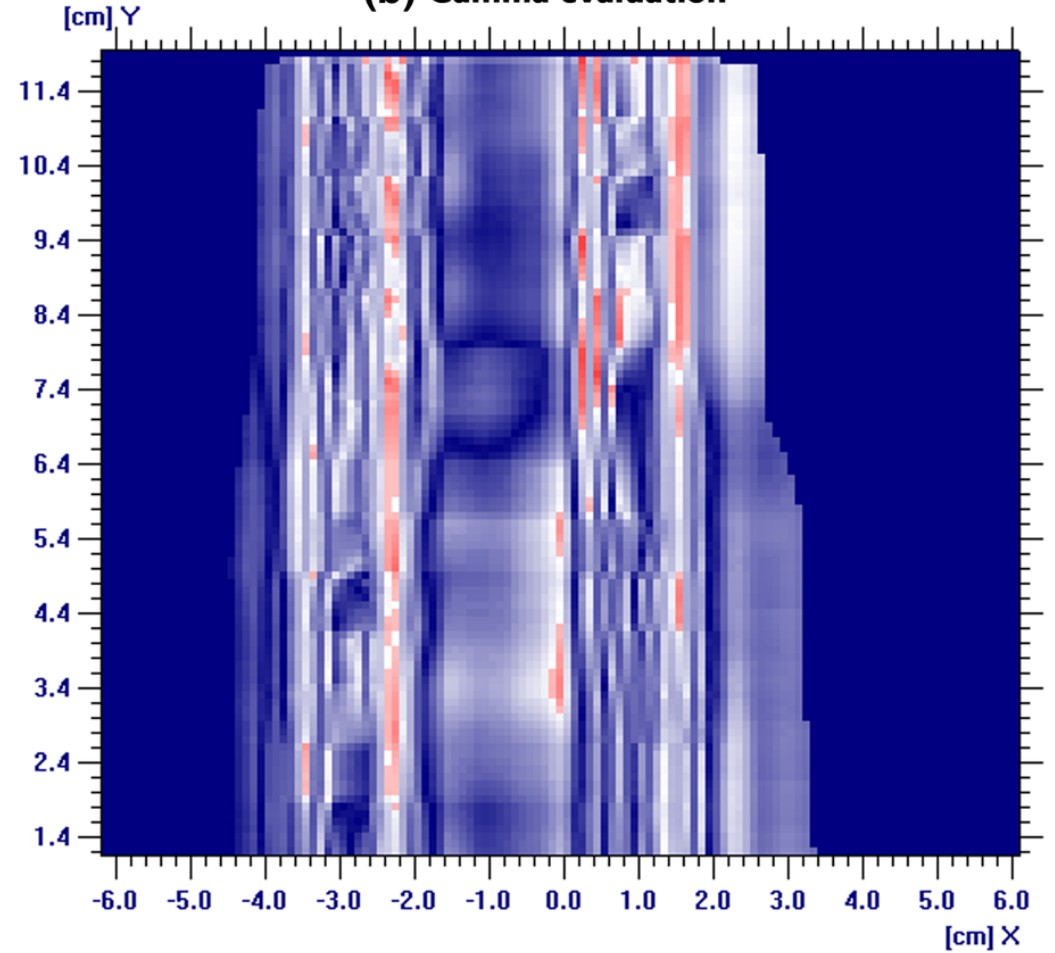

(c) Spinal junction profile

120.0 [\%]Signal

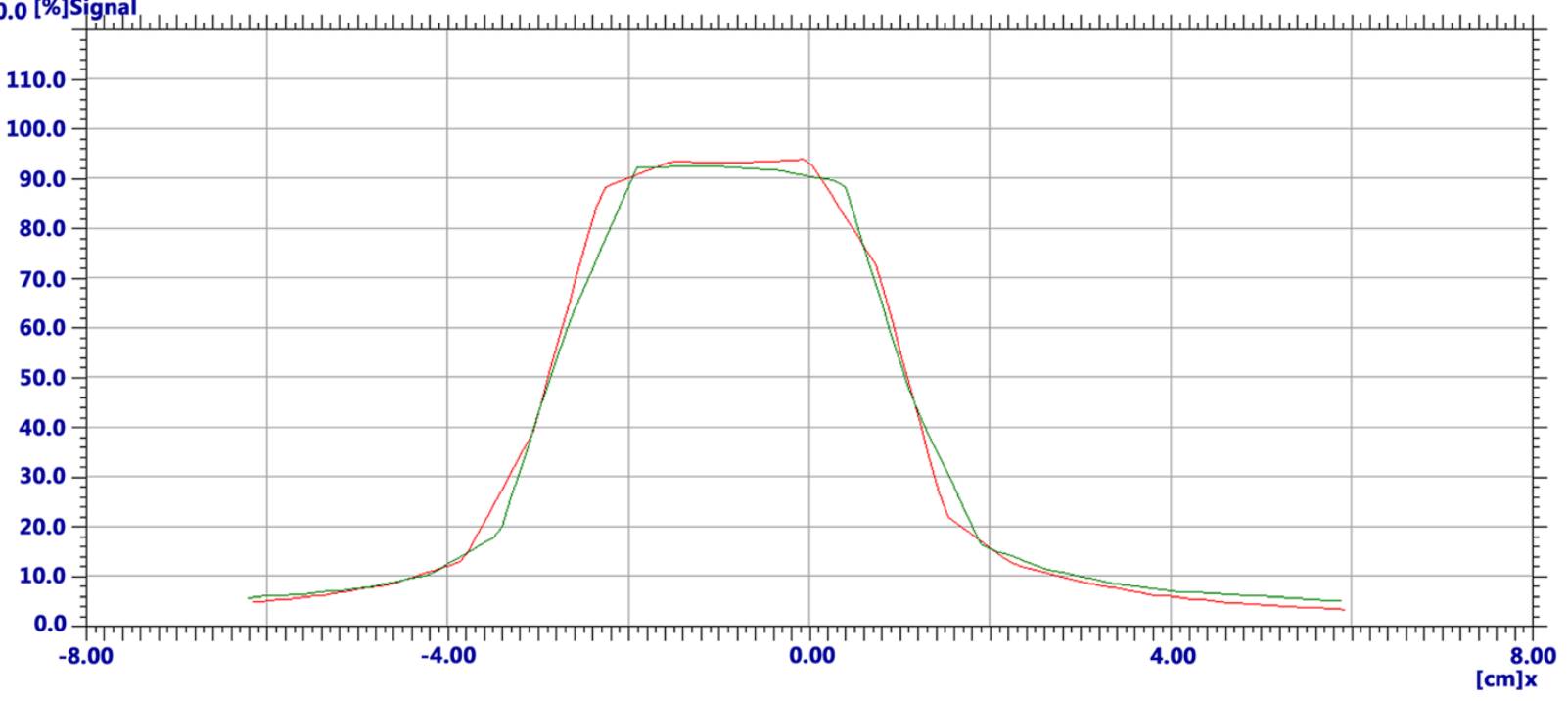

Figure 5 QA results in the overlapping spinal cord region with TIOJ plan for patient $\mathbf{5}$. (a) and (c) show the coronal plane dose

distributions and profiles for the spinal-spinal junction. The black lines indicate the junction regions. (b) shows the gamma index (3\% for absolute dose and $3 \mathrm{~mm}$ ) for the relative dose evaluation.

spots in beam overlapping areas. And with the new TIOJ technique, the lengths of all radiation fields have the maximum length set by MLC $(40 \mathrm{~cm})$. In TIJJ, field lengths are manually set. In contrast, larger beam overlap regions can be achieved in the target area with TIOJ, and the planning process involves far less manual setting and adjustment which facilitates dose optimization in the inverse planning system and helps avoid dose cold spots and hot spots in beam overlap regions.

In the TIJJ plan proposed by Cao et al. [9], the filed length in the overlap region between isocenters needs to be manually adjusted in order to generate jagged 
junction areas with $1.1 \mathrm{~cm}$ intervals. During this process, the field width in left-right direction also has to be manually adjusted to avoid excessive radiation field width relative to patient lateral size and MLC leakage. The planning process is complex and time consuming. The proposed TIOJ method only requires three isocenters to be manually determined. The field size and shape are then automatically set by the planning system. The new method greatly simplifies the planning process and reduces planning time. The average time needed to make a TIOJ IMRT plan is about 10 min shorter than that using TIJJ (30 min vs. $40 \mathrm{~min}$ ). This simplification can also facilitate a standardized design procedure, which minimizes human errors in the process.

VMAT, another emerging radiation therapy technology, has also been applied in CSI treatment $[4,5]$. With its high dose rate and technical advantages similar to helical tomotherapy, VMAT can satisfactorily solve some CSI related problems (such as a long target area and matching between radiation fields). Lee et al. [5] applied VMAT technology to treat five patients. PTV lengths were $48.1-83.7 \mathrm{~cm}$. The prescription dose was 23.4Gy in13 fractions. The final mean CI and HI were 1.22 (range: 1.09-1.45) and 1.04 (range: 1.03-1.07), respectively. The formulas Lee et al. used to calculate $\mathrm{HI}$ and CI were different from ours. For comparison purposes, we used their formulas to re-process the data collected from our nine patients. The $\mathrm{CI}$ and $\mathrm{HI}$ found with the TIOJ method were 1.23 (range: 1.17-1.34), and 1.08 (range: 1.07-1.11), respectively. Although we could not perform statistical analyses to compare their data with ours, it can be seen that the CI and HI obtained using TIOJ for CSI were very similar to that obtained by Lee et al. using the VMAT technique.

Most patients that receive CSI treatment are teenagers. Before treatment, evaluation of OAR dose needs to be performed to minimize the incidence of radiotherapyrelated complications. In the current study, dose limitation requirements were defined for the lens and eyes, similar to the studies by Cao et al. [9] and Lee et al. [5]. Figure 4 shows the OAR doses received with each of the two plans, OAR doses were at a satisfactory level and basically the same with TIOJ and TIJJ. The mean dose of left lens and right lens was 6.89 and 6.91Gy, respectively, using TIOJ. The mean dose of left lens and right lens was 6.91 and 6.78GY, respectively, using TIJJ. These doses were significantly lower than the 9.1Gy, as reported by Cao et al. [9], and significantly lower than the 20Gy and 18Gy reported by Lee et al. [5] with VMAT. These differences may be due to the more stringent limit conditions we set for the lens and eye.

The total MU and the mean dose received by the torso are the most important risk factors for secondary tumors [14-16]. We found that the total MU and dose in the torso using TIOJ were similar to that found using TIJJ. Total MU using TIOJ was 1907.4 \pm 60.6 (range: 1575 2104). Total MU using TIJJ was $1903.3 \pm 34.8$ (range: 1575-2253). So when use the same dose rate, the deliver (beam on) time for both technique (TIOJ and TIJJ) will be very similar. We also found that the total MU were substantially higher than that reported by Lee et al. with VMAT. This suggests that, VMAT technology may be more advantageous than IMRT in reducing the incidence of secondary tumors. We believe that as IMRT treatment involves a large amount of MLC occlusion, total MU may not fully reflect dose received by the whole body. In the current study, mean dose received by the torso was calculated as $8.75 \pm 0.59$ with TIOJ and $8.26 \pm 0.53$ with TIJJ. There was no significant difference between the two plans. As Lee et al. did not calculate the torso dose with VMAT, we were not able to make relevant comparisons.

\section{Conclusions}

The TIOJ IMRT method for CSI treatment outlined in this article can creat plans with satisfactory $\mathrm{CI}$ and $\mathrm{HI}$. The use of three isocenters and beam overlap regions between the isocenters helps avoid typical CSI problems, such as over-long radiation fields and matching between the fields. As compared to previously reported methods, TIOJ greatly simplified the steps required to manually set field widths and boundaries and improved efficiency. Only one treatment plan and a simple bed capable of moving in one direction are needed to complete the entire treatment. TIOJ IMRT provides a simple and efficient choice for CSI treatment.

\section{Abbreviations \\ CSI: Craniospinal irradiation; TIOJ: Three-isocenter overlap-junction; TIJJ: Three-isocenter jagged-junction; HI: Heterogeneity index; Cl: Conformity index; OARs: Organs at risk; 3DCRT: Three-dimensional conformal radiotherapy; IMRT: Intensity modulated radiation therapy; VMAT: Volumetric modulated arc therapy; PTV: Planning target volume; MLC: Multileaf collimator; SAD: Source to axis; DVH: Dose volume histogram; ICRU: International commission on radiation units; MUs: Monitor units; SSD: Source to surface distances.}

\section{Competing interests}

The authors declare that they have no competing interests.

\section{Authors' contributions}

WZ, JW and FYM participated in the study design, contributed equally in analysis of data and drafting the manuscript, GY, CZ and SB participated in the treatment planning. GY made important contributions in collecting and analyzing data, and in revising the content. All authors read and approved the final manuscript.

\section{Author details}

'Department of Radiation Oncology, Huanhu Hospital, Tianjin, China. ${ }^{2}$ Department of Radiation Oncology, Tianjin Medical University Cancer Hospital, Tianjin, China. ${ }^{3}$ Department of Biomedical Engineering, Tianjin University, Tianjin, China.

Received: 13 May 2013 Accepted: 15 September 2013 Published: 17 September 2013 


\section{References}

1. Bauman G, Yartsev S, Coad T, Fisher B, Kron T: Helical tomotherapy for craniospinal radiation. Br J Radiol 2005, 78:548-552. PMID:15900062.

2. Parker W, Brodeur M, Roberge D, Freeman C: Standard and nonstandard craniospinal radiotherapy using helical tomoTherapy. Int J Radiat Oncol Biol Phys 2010, 77:926-931. PMID:20231076.

3. St.Clair WH, Adams JA, Bues M, Fullerton BC, La Shell S, Kooy HM, Loeffler JS, Tarbell NJ: Advantage of protons compared to conventional X-ray or IMRT in the treament of a pediatric patient with medulloblastoma. Int J Radiat Oncol Biol Phys 2004, 58:727-734. PMID:14987427.

4. Fogliata A, Bergstrom S, Cafaro I, Clivio A, Cozzi L, Dipasquale G, Hållström P, Mancosu P, Navarria P, Nicolini G, Parietti E, Pesce GA, Richetti A, Scorsetti

$M$, Vanetti $E$, Weber $D C$ : Cranio-spinal irradiation with volumetric modulated arc therapy: a multi-institutional treatment experience. Radiother Oncol 2011, 99:79-85. PMID:21421273.

5. Lee YK, Brooks CJ, Bedford JL, Warrington AP, Saran FH: Development and evaluation of multiple isocentric volumetric modulated arc therapy technique for craniospinal axis radiotherapy planning. Int J Radiat Oncol Biol Phys 2012, 82:1006-1012. PMID:21345612.

6. Seppala J, Kulmala J, Lindholm P, Minn H: A method to improve target dose homogeneity of craniospinal irradiation using dynamic split field IMRT. Radiother Oncol 2010, 96:209-215. PMID:20581696.

7. Pai Panandiker A, Ning H, Likhacheva A, Ullman K, Arora B, Ondos J, Karimpour S, Packer R, Miller R, Citrin D: Craniospinal irradiation with spinal IMRT to improve target homogeneity. Int I Radiat Oncol Biol Phys 2007, 68:1402-1409. PMID:17467921.

8. Parker WA, Freeman CR: A simple technique for craniospinal radiotherapy in the supine position. Radiother Oncol 2006, 78:217-222. PMID:16330119.

9. Cao F, Ramaseshan R, Corns R, Harrop S, Nuraney N, Steiner P, Aldridge S, Liu M, Carolan H, Agranovich A, Karvat A: A three-isocenter jaggedjunction IMRT approach for craniospinal irradiation without beam edge matching for field junctions. Int I Radiat Oncol Biol Phys 2012, 84:648-654 PMID:22436794.

10. International Commission of Radiation Units and Measurements: ICRU Report 50: Prescribing, recording, and reporting photon beam therapy. Bethesda: International Commission on Radiation Units and Measurements; 1993.

11. International Commission of Radiation Units and Measurements: ICRU Report 62: Prescribing, recording, and reporting photon beam therapy (supplement to ICRU Report 50). Bethesda: International Commission on Radiation Units and Measurements; 1999

12. Kusters JM, Louwe RJ, van Kollenburg PG, Kunze-Busch MC, Gidding $C E$, van Lindert EJ, Kaanders JH, Janssens GO: Optimal normal tissue sparing in craniospinal axis irradiation using IMRT with daily intrafractionally modulated junction. Int I Radiat Oncol Biol Phys 2011, 81:1405-1414. PMID:21300472.

13. Parker W, Filion E, Roberge D, Freeman CR: Intensity-modulated radiotherapy for craniospinal irradiation: target volume considerations, dose constraints, and competing risks. Int I Radiat Oncol Biol Phys 2007, 69:251-257. PMID:17707279.

14. Followill D, Geis P, Boyer A: Estimates of whole-body dose equivalent produced by beam intensitymodulated conformal therapy. Int I Radiat Oncol Biol Phys 1997, 38:667-672. PMID:9231693.

15. Hall EJ: Intensity-modulated radiation therapy, protons, and the risk of second cancers. Int J Radiat Oncol Biol Phys 2006, 65:1-7. PMID:16618572.

16. Kry SF, Salehpour M, Followill DS, Stovall M, Kuban DA, White RA, Rosen II: The calculated risk of fatal secondary malignancies from intensity modulated radiation therapy. Int I Radiat Oncol Biol Phys 2005, 62:1195-1203. PMID:15990025.

doi:10.1186/1748-717X-8-217

Cite this article as: Wang et al:: A simple approach of three-isocenter IMRT planning for craniospinal irradiation. Radiation Oncology 2013 8:217.

\section{Submit your next manuscript to BioMed Central and take full advantage of:}

- Convenient online submission

- Thorough peer review

- No space constraints or color figure charges

- Immediate publication on acceptance

- Inclusion in PubMed, CAS, Scopus and Google Scholar

- Research which is freely available for redistribution
C Biomed Central 